\title{
Céréaliculture commerciale et concentration
} foncière

Un cas d'étude dans les terres basses du Sud-Veracruz, Mexique Commercial cereal production and land concentration. A case study in the lowlands of South-Veracruz.

\section{Virginie Brun}

\section{OpenEdition}

\section{Journals}

Édition électronique

URL : http://journals.openedition.org/transcontinentales/1107

DOI : $10.4000 /$ transcontinentales. 1107

ISBN : 978-2-7351-1572-3

ISSN : 1775-397X

\section{Éditeur}

Editions de la maison des sciences de l'homme

Référence électronique

Virginie Brun, "Céréaliculture commerciale et concentration foncière », Transcontinentales [En ligne] 10/11 | 2011, document 3, mis en ligne le 19 octobre 2011, consulté le 08 septembre 2020. URL : http://journals.openedition.org/transcontinentales/1107 ; DOI : https://doi.org/10.4000/ transcontinentales. 1107

Ce document a été généré automatiquement le 8 septembre 2020.

Tous droits réservés 


\title{
Céréaliculture commerciale et concentration foncière
}

\author{
Un cas d'étude dans les terres basses du Sud-Veracruz, Mexique \\ Commercial cereal production and land concentration. A case study in the \\ lowlands of South-Veracruz.
}

\section{Virginie Brun}

\section{Introduction}

1 La multiplication récente des cas d'appropriations foncières dans les pays du Sud a replacé la question de la sécurité alimentaire et du devenir des agricultures familiales au cœur des débats internationaux sur le développement ${ }^{1}$. Au-delà des questions éthiques que les exemples les plus médiatisés ont suscitées au sein de l'opinion publique, ces phénomènes permettent d'éclairer d'un jour nouveau les transformations productives en cours dans de nombreuses régions agricoles du monde, beaucoup moins spectaculaires mais tout autant significatives du point de vue de leurs impacts sur le développement des agricultures familiales et sur l'accès de celles-ci aux ressources productives, notamment foncières. Ces nouvelles tensions foncières amènent également à questionner les formes de production et d'intégration qui accompagnent l'avènement de l'agrobusiness comme modèle de développement, parfois en lien étroit avec les mouvements d'appropriation de terres ${ }^{2}$.

Dans cet article, nous tenterons d'illustrer ces dynamiques à travers l'analyse des recompositions productives dans deux petites régions des terres basses du SudVeracruz, au Mexique, où la céréaliculture moto-mécanisée connaît un fort développement depuis une quinzaine d'années. Ces transformations s'inscrivent dans un processus d'ouverture des marchés et de restructuration du secteur agricole mexicain, engagé dans la seconde moitié des années 1980 et connaissant une forte accélération depuis l'entrée en vigueur de l'Accord de libre-échange nord-américain (ALENA) signé, en 1994, entre le Mexique, les États-Unis et le Canada. Dans les régions 
et les bassins agricoles à fort potentiel de développement, le retrait de l'État de la sphère productive profite de façon générale au secteur privé (agro-industriels, entrepreneurs agricoles, fournisseurs d'intrants et de services agricoles, grands éleveurs) qui s'est rapidement positionné comme promoteur et accompagnateur du changement. C'est ce que l'on observe actuellement dans certains ejidos ${ }^{3}$ des terres basses du Sud-Veracruz. La libération des institutions ejidales vis-à-vis du pouvoir politique central et le changement institutionnel promu autour du foncier ont permis, quant à eux, l'ouverture d'un espace nouveau pour les initiatives privées et individuelles au sein des ejidos. Ceci est reflété notamment par l'émergence d'une catégorie d'ejidataires capitalisés développant des liens étroits avec le secteur privé et l'industrie céréalière. La «modernisation" et l'intensification des systèmes de production céréaliers attirent par ailleurs l'intérêt croissant de nouveaux investisseurs, dont la présence dans les terres basses a des effets notables sur les dynamiques foncières et sur l'accélération des phénomènes de concentration de terres ${ }^{4}$.

3 Après une petite décennie marquée par d'importants changements techniques et institutionnels dans les ejidos - par exemple, moto-mécanisation des systèmes de culture, intensification productive par l'usage croissant d'engrais et de produits phytosanitaires d'origine industrielle, amélioration de l'accès au crédit agricole, intégration commerciale des exploitations familiales -, l'achèvement du processus de déboisement et de mise en valeur des terres agricoles dans ces deux régions a conduit à un recentrage de la problématique de développement autour de l'accès au foncier. La " course à la terre " prend d'autant plus d'ampleur que le processus de mécanisation des systèmes de production agricole s'accélère et que le désenclavement récent de ces zones fertiles encourage les stratégies de croissance foncière, et encore plus, depuis la hausse récente du prix des matières premières agricoles.

Dans la première partie, nous présentons les processus productifs à l'œuvre dans ces deux régions en les replaçant dans le contexte des réformes néolibérales et des modifications apportées au cadre législatif agraire au Mexique. Nous analysons en particulier les incidences des changements sur les dynamiques foncières au sein des ejidos. Dans une deuxième partie, nous nous intéressons plus particulièrement au rôle joué par le secteur privé (investisseurs, entrepreneurs, propriétaires fonciers, etc.) dans l'évolution de ces dynamiques et aux dispositifs mis en place pour contrôler la production d'une part, et l'accès des petites exploitations familiales aux ressources productives, notamment foncières, d'autre part. Nous nous interrogeons enfin sur les conséquences des nouvelles formes de production qui se mettent en place dans ces deux régions. Qu'ils renvoient à des schémas proches de ceux initiés par l'agrobusiness (agriculture de contrat) ou à des systèmes fonctionnant sur des bases clientélistes, ces dispositifs se traduisent par une concentration accrue des terres aux mains des détenteurs du capital technique et financier mais, aussi, par une perte de contrôle du processus de production pour les petits agriculteurs. Ceci soulève la question de la souveraineté de ces derniers dans leurs choix et leurs stratégies productives. 


\section{Transformations productives et évolution de la Problématique foncière}

\section{Des changements qui interviennent dans un contexte de réformes et de libéralisation des marchés}

Définies à la fin des années 1980, les réformes néolibérales promues par le gouvernement mexicain dans le domaine agricole avaient pour principal objectif d'augmenter la production et la productivité du secteur en forçant la réallocation des ressources productives vers les structures les plus "efficientes" et les plus compétitives dans le nouveau jeu du libre-échange. La modification de l'article 27 de la Constitution, en 1991, a constitué le pilier central de ces réformes : premièrement, en proclamant la fin de la réforme agraire après plus de 70 ans de redistributions foncières et, deuxièmement, en posant les bases d'une privatisation des ejidos à travers la légalisation des marchés fonciers sur les terres ejidales. Pour accompagner cette réforme, un important programme d'inscription au cadastre et de titularisation des droits fonciers - de type individuel et privatif - a été lancé à grands renforts de moyens dans l'ensemble des ejidos et des communautés agraires du pays (PROCEDE ${ }^{5}$ ). Il a abouti, à ce jour, à la délivrance de certificats individuels de droit agraire dans $89 \%$ des ejidos du pays ${ }^{6}$.

6 L'État a par ailleurs procédé à la liquidation et à la privatisation des entreprises parapubliques, jusqu'alors chargées d'encadrer et de contrôler la production agricole, laissant au secteur privé le soin d'encourager et d'accompagner les changements nécessaires à la recomposition du secteur. Dans cette optique, les modalités de l'intervention publique ont été entièrement redéfinies, selon une segmentation thématique accrue des programmes et un ciblage précis des différentes catégories de producteurs : les grandes zones d'agriculture commerciale ont ainsi reçu l'essentiel des fonds alloués au titre des programmes de soutien à la production, tandis que les régions agricoles marginalisées sont devenues la cible de programmes sociaux de traitement de la pauvreté. Ceci a eu pour effet d'accroître fortement les inégalités intrasectorielles et interrégionales de développement ${ }^{7}$.

7 Dans les zones de petite agriculture commerciale et les régions à fort potentiel de développement, l'ensemble de ces réformes s'est traduit par d'importantes restructurations au sein des filières locales, dans lesquelles le secteur privé semble avoir largement trouvé sa place, tant en amont (agrofournitures) qu'en aval (mise en marché) de la production. On l'observe actuellement dans certains ejidos des terres basses du Sud-Veracruz, où de nouveaux acteurs issus du secteur privé participent, depuis le milieu des années 1990, au développement d'une petite céréaliculture familiale à vocation commerciale. Petits entrepreneurs régionaux, entreprises agroindustrielles, et même grands éleveurs apparaissent de fait comme des acteurs incontournables dans les restructurations locales en cours.

Les processus productifs à l'œuvre : moto-mécanisation des systèmes céréaliers

9 Les deux sous-régions d'étude sont situées dans la plaine littorale du Sotavento, au sud de l'État de Veracruz. Il s'agit de deux plaines alluviales de basse altitude, communément appelées terres basses, soumises à de fortes crues annuelles du fleuve Tesechoacan, dans un cas, et du fleuve Coatzacoalcos, dans l'autre (Fig. 1). Les terres 
basses du Tesechoacan et du moyen-Coatzacoalcos présentent des caractéristiques hydromorphologiques (terres inondables) et agraires (prédominance du secteur ejidal) similaires. Depuis le milieu des années 1990, on observe dans les ejidos de ces deux régions des transformations productives majeures qui s'inscrivent dans un processus global de transition des exploitations familiales d'une agriculture tournée vers l'autoconsommation à une céréaliculture à vocation commerciale. Les changements touchent autant les pratiques et les conduites culturales (mécanisation, intensification des systèmes de culture, monoculture, etc.) que les dispositifs de mise en relations des producteurs avec les marchés. Si les processus à l'œuvre dans les deux zones présentent des similitudes, notamment en termes d'intensification et de changements techniques, ils s'inscrivent toutefois dans des contextes locaux très différents, qui renvoient à des trajectoires historiques de différenciation des systèmes de production distinctes mais aussi à des stratégies d'acteurs différentes.

Figure 1

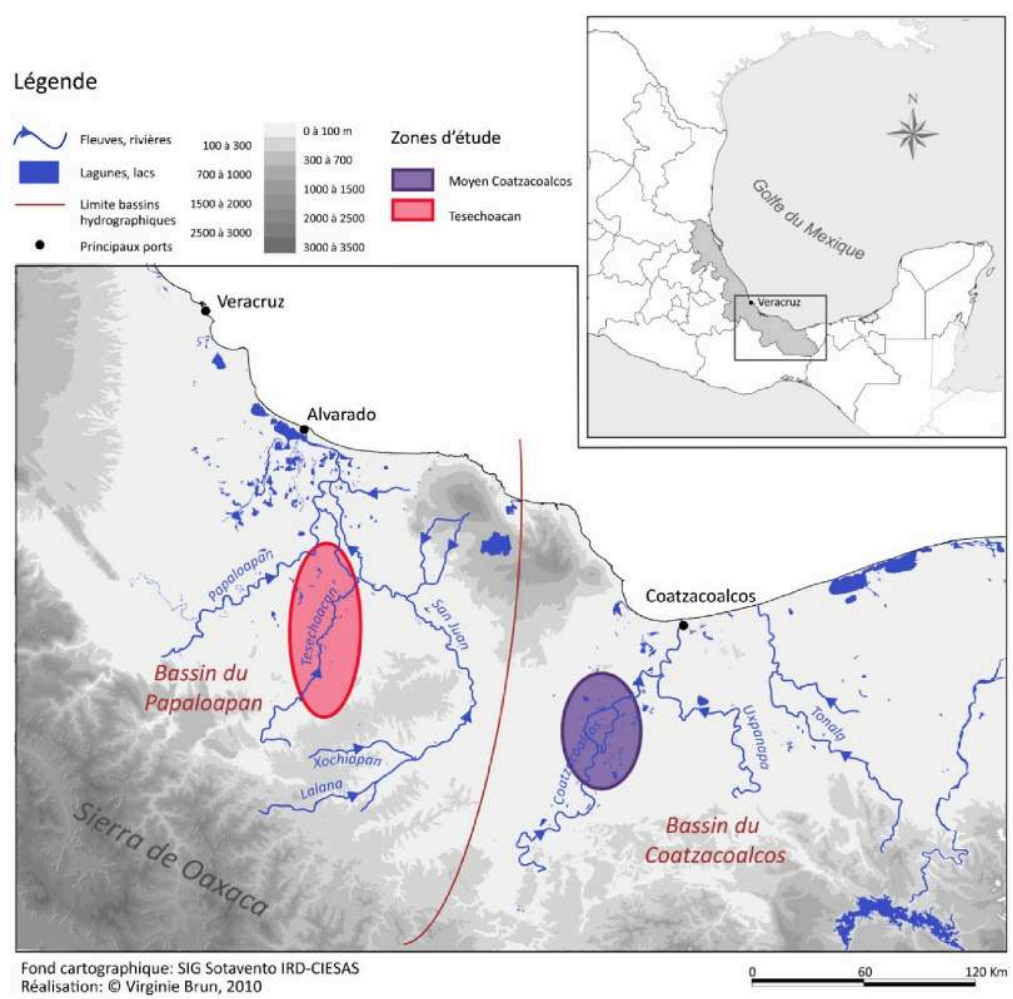

La région d'étude

Fond cartographique SIG Sotavento IRD-CIESAS. Réalisation (c) Virginie Brun, 2010

\section{Le cas du Tesechoacan}

Les transformations intervenues dans les terres basses du Tesechoacan concernent principalement la culture de maïs blanc destiné à la fabrication industrielle de farine pour l'alimentation humaine. En termes de changements techniques, la motomécanisation de l'ensemble des travaux agricoles ainsi que l'utilisation de semences commerciales et d'intrants d'origine industrielle (engrais, pesticides, etc.) se sont généralisées en moins de dix ans, ce qui a permis une hausse spectaculaire des rendements agricoles : 3 tonnes à l'hectare en moyenne, en 1995, contre 7 à $8 \mathrm{t} / \mathrm{ha}$ 
aujourd'hui sur l'ensemble des ejidos de la région. La mécanisation du semis et l'utilisation de moissonneuses-batteuses pour la récolte ont eu pour effet une augmentation rapide des superficies semées et des quantités de maïs commercialisées.

Le processus d'intensification des systèmes céréaliers, amorcé dans le courant des années 1980, a connu une forte accélération, au début des années 1990, dans le cadre de la nouvelle politique agricole mexicaine, dont un des programmes visait la «modernisation » des exploitations ejidales situées dans les zones à fort potentiel de développement ${ }^{8}$. Le dispositif d'encadrement et d'accompagnement prévu dans le cadre de ce programme est appuyé en grande partie sur le secteur privé, à qui l'État avait assigné le rôle de relais institutionnel, technique et financier au niveau local ${ }^{9}$. Dans le Tesechoacan, c'est une petite entreprise régionale d'agro-services qui a tenu ce rôle, axant son intervention dans les ejidos sur deux points principaux: la diffusion de "paquets technologiques " à crédit, contraignant de fait les agriculteurs à adopter le semis direct mécanisé, et l'intégration commerciale des exploitations ejidales à travers la création d'une organisation de producteurs. Dans un premier temps ciblé sur les producteurs les plus solides financièrement et disposant de matériel agricole (soit en propriété, soit par le biais de la location), le dispositif s'est peu à peu étendu aux autres ejidataires. Mais, l'ouverture du crédit à toutes les catégories de producteurs n'a été effective qu'avec l'intégration des exploitations ejidales selon un système proche de celui de l'agriculture contractuelle : une même entreprise fournit les intrants à crédit et achète la récolte, ce qui lui permet de réduire les risques d'impayés et par conséquent d'ouvrir le crédit jusqu'aux producteurs les moins solvables.

\section{Le cas du Moyen-Coatzacoalcos}

Dans le cas du Moyen-Coatzacoalcos, la mécanisation et l'intensification des systèmes céréaliers ont, dans un premier temps, concerné le sorgho destiné à l'alimentation animale avant de s'étendre au maïs. Contrairement à ce qui s'est produit dans le Tesechoacan, l'État n'a ici joué aucun rôle direct dans l'initiation du processus. Jusqu'à des dates très récentes, les ejidos des terres basses du Moyen-Coatzacoalcos ont rarement bénéficié de programmes publics de soutien à la production agricole sur le modèle du Pronamat. Les contraintes physiques du milieu, dues à un réseau hydrographique beaucoup plus dense que dans le Tesechoacan, et l'enclavement de cette zone, du fait du manque de routes et de ponts, peuvent en partie l'expliquer. Mais, la force des réseaux de pouvoir et des systèmes clientélistes contrôlés historiquement par les grands éleveurs de la région explique la présence limitée de l'État dans les terres basses du Moyen-Coatzacoalcos. De fait, la transformation des systèmes de culture dans les ejidos est essentiellement due à l'intervention de ces grands éleveurs.

13 Ces derniers, contraints, au milieu des années 1990, en plein contexte de crise de l'élevage bovin extensif, de diversifier leurs investissements et de s'adapter à l'évolution de la filière d'embouche (perte de valeur des animaux finis à l'herbe au profit d'animaux finis en parcs d'engraissement), se sont en effet lancés dans la production de céréales. Les terres basses du Moyen-Coatzacoalcos, alors faiblement mises en valeur autrement que par l'élevage, ont rapidement attiré leur convoitise. En quelques années, les acquisitions foncières pour la production de sorgho se sont multipliées. Puis, la céréaliculture s'est diffusée au sein des ejidos, sur la base de relations de type clientéliste. On a ainsi assisté à une intégration économique complète des ejidos du Moyen-Coatzacoalcos autour des activités agricoles impulsées et 
contrôlées par les grands éleveurs : élevage, bien entendu, mais aussi céréaliculture commerciale. Un contrôle qui s'exerce autant en amont, pour l'accès aux ressources productives (intrants, matériel agricole) qu'en aval, pour la mise en marché des productions.

\section{Les incidences foncières du changement dans les ejidos}

La restructuration des filières locales et l'intensification des systèmes de culture dans les ejidos des terres basses ont eu plusieurs effets notables en termes d'utilisation du foncier. En premier lieu, la mécanisation du semis et l'amélioration de l'accès aux ressources productives, que ce soit par le biais du crédit dans le Tesechoacan ou des réseaux établis par les grands éleveurs dans le Coatzacoalcos, ont permis une valorisation des terres arables sans précédent sur l'ensemble des superficies ejidales, comme en témoignent les changements très nets observés dans le paysage : en moins de dix ans, le défrichement et le dessouchage systématiques des parcelles ont rendu apte au travail mécanisé la quasi-totalité des terres arables disponibles dans les ejidos. Par ailleurs, l'épuisement des réserves foncières a rapidement fait surgir un problème de limite physique à l'amélioration des systèmes de culture. Ceci, combiné à un ensemble d'autres facteurs, a eu pour effet d'augmenter de façon très nette la demande sur les marchés fonciers locatifs :

Premièrement, l'augmentation de la productivité permise par la mécanisation a libéré du temps de travail pour la main-d'œuvre familiale, temps qui est, dans la mesure du possible, mis à profit pour agrandir les superficies semées.

Deuxièmement, la mise en place d'itinéraires techniques fortement mécanisés a incité les détenteurs de matériel agricole à augmenter les superficies semées pour abaisser le coût marginal de l'amortissement, ce qui s'est traduit par une demande très forte de la part de cette catégorie de producteurs sur les marchés locatifs.

17 Troisièmement, l'amélioration de l'accès aux ressources productives autres que la terre a permis de minimiser les débours monétaires en début de cycle et de constituer parfois une réserve d'argent pour la prise de terres en faire-valoir indirect.

Dans un contexte où il y a une grande disponibilité de matériel agricole, notamment de tracteurs, et où le marché de la location fonctionne plutôt bien, l'enjeu productif dans les terres basses se concentre donc progressivement sur l'accès à la terre et sur des stratégies de croissance foncière des exploitations ejidales. Cela provoque une augmentation de la demande sur les marchés fonciers, mais aussi une accélération du processus de différenciation interne aux ejidos, entre les ejidataires qui possèdent l'équipement agricole d'une part, et ceux pour qui le recours à la location de matériel constitue une limite à l'agrandissement des superficies semées d'autre part. Toutefois, la situation de déséquilibre entre l'offre et la demande de terres au sein des ejidos tend à une extension géographique du phénomène au-delà des limites territoriales de l'ejido, et au rapprochement des ejidataires avec les différents propriétaires privés des terres basses. L'arrivée de nouveaux acteurs et investisseurs privés ouvrent, à ce titre, des perspectives de croissance pour les ejidataires les mieux dotés, qui prennent toutefois des caractères différents selon que le rapprochement se fait sur la base de relations contractuelles, formelles ou de dépendance. 


\section{Secteur privé, concentration foncière et contrôle de l'accès aux ressources productives}

\section{Un nouveau cadre législatif qui limite la propriété mais ouvre la voie à la concentration foncière}

En clarifiant les droits agraires individuels et en garantissant la sécurité foncière, le PROCEDE devait, en principe, contribuer directement au développement des marchés fonciers dans les ejidos. Dans les faits, ni le PROCEDE ni la légalisation des transactions foncières n'ont fondamentalement changé la donne en matière de distribution et de circulation de la terre au sein des ejidos. Il y a deux raisons principales à cela: premièrement, les ejidos avaient pour la plupart déjà procédé de manière interne à une individualisation des droits d'usage (connue comme le parcelamiento económico) et, deuxièmement, les transactions foncières étaient monnaie courante en dépit de leur interdiction, jusqu'en 1991. Le parcelamiento económico avait donc déjà permis une reconnaissance individuelle des droits d'usage - bien que non officielle - et chaque ejidataire jouissait tacitement d'une relative sécurité foncière. Par ailleurs, l'autorisation des transferts de terre par cession temporaire (fermage, métayage, prêt) ou définitive (achat/vente) a surtout permis de légaliser des pratiques déjà largement répandues. Plusieurs études de cas réalisées au Mexique dressent des conclusions similaires qui tendent à minimiser les effets de la libéralisation des marchés fonciers sur l'accélération des transferts de terre au sein des ejidos ${ }^{10}$.

En revanche, la réforme de 1991 a fait sauter l'un des verrous mis en place au lendemain de la révolution mexicaine de 1910 pour limiter la concentration foncière : tout en réaffirmant le caractère hors la loi de la grande propriété foncière et en conservant les seuils limites d'extension de la propriété privée, la réforme constitutionnelle a permis d'ouvrir l'accès au foncier rural aux sociétés par actions, ce qui était jusqu'alors interdit. L'acquisition des terres agricoles ou forestières peut désormais se faire à condition que la superficie moyenne détenue par chaque actionnaire ne dépasse pas le seuil légal de la propriété privée individuelle et que la superficie totale n'excède pas vingt-cinq fois ce seuil. Mais ce qui vaut pour les acquisitions de type achat/vente ne s'applique pas à la location foncière. Rien n'empêche donc une société privée ou un exploitant agricole de louer la totalité d'un ou plusieurs ejidos, puisque la loi ne prévoit aucune limite à l'accumulation foncière par la voie de la location. Dès lors, il est possible à toute personne physique ou morale de contrôler plusieurs milliers d'hectares en toute légalité.

\section{Des acquisitions foncières qui s'opèrent principalement dans le secteur de la propriété privée}

21 D'une manière générale, on constate peu de ventes de terres au sein des ejidos et, lorsque des transactions se concrétisent, elles impliquent le plus souvent des membres d'un même ejido ${ }^{11}$. De rares cas d'achats de terres par des personnes extérieures ont pu être observés dans certains ejidos du Moyen-Coatzacoalcos; ils concernent des parcelles situées dans des zones particulièrement marécageuses et, de fait, peu ou pas mises en valeur, qui nécessitent généralement des travaux d'aménagement foncier pour les rendre aptes à la mécanisation : drainage, dessouchage dans les zones boisées, etc. 
La propriété privée connaît en revanche un marché foncier un peu plus dynamique. Les domaines mis en vente ne concernent souvent pas moins de plusieurs centaines d'hectares, ce qui exclut d'office la participation des ejidataires sur ce marché. Les transferts s'effectuent donc principalement entre propriétaires privés. Les propriétés vendues appartiennent le plus souvent à des agriculteurs ou éleveurs de taille moyenne, sans repreneur au sein de leur famille ou ne disposant pas de capitaux suffisants pour investir dans l'amélioration de leur exploitation (achat de matériel agricole, aménagements fonciers, etc.). Les acheteurs de terres sont quant à eux de plusieurs types : dans le Tesechoacan, ce sont surtout des agro-industriels du secteur céréalier qui détiennent un capital fixe d'exploitation conséquent et possèdent leur propre réseau de commercialisation et de transformation; on trouve également quelques exploitants agricoles et petits négociants locaux qui profitent de la dynamique créée autour de la céréaliculture pour diversifier leurs activités. Dans le Moyen-Coatzacoalcos, les acheteurs sont principalement des grands éleveurs déjà propriétaires de plusieurs ranchos, qui acquièrent des terres pour produire du sorgho (et du maïs dans une moindre mesure). Comme dans le Tesechoacan, on trouve également quelques commerçants et revendeurs d'intrants agricoles installés dans les villes voisines.

Dans tous les cas, les stratégies d'acquisition foncière répondent clairement à des logiques économiques, qui passent par une extension des activités agricoles pour les uns (cas des éleveurs ou des agro-industriels) ou par une diversification des activités pour les autres (cas des commerçants). Les acquisitions sont de taille variable, de plusieurs centaines à plusieurs milliers d'hectares.

\section{Des logiques de croissance qui reposent sur l'intégration et le contrôle des exploitations familiales}

24 Dans la mesure du possible, les nouveaux propriétaires fonciers optent pour un mode d'exploitation en faire-valoir direct, avec emploi de main-d'œuvre salariée. Cependant, quand le contrôle sur la main-d'œuvre et sur l'équipement agricole devient trop coûteux, l'externalisation de certaines activités agricoles devient en général la règle, selon des arrangements agraires à mi-chemin entre la cession en fermage et la prestation de service.

Dans un des cas étudiés - 300 hectares -, l'agro-industriel propriétaire cède en location 200 ha à un ejidataire dans le cadre d'un contrat de type fermage: la rente foncière, payable après récolte, est fixée à 600 pesos/ha (soit un peu plus de la moitié du prix actuel de la terre sur le marché locatif) ; les intrants agricoles sont fournis à crédit par l'agro-industriel, qui possède son entreprise de distribution d'intrants ; l'ejidataire conserve dans ce cas $100 \%$ du produit brut de la récolte. Sur les 100 ha restants, l'ejidataire fournit une "prestation de service" selon l'arrangement suivant: le propriétaire foncier prend à sa charge l'intégralité du coût des intrants et conserve $100 \%$ du produit brut de la récolte; les travaux effectués par l'ejidataire sont rémunérés à leur valeur de marché. Au final, le coût de la location et le coût des intrants sur les 200 ha sont déduits du coût des travaux engagés par l'ejidataire sur les 100 ha, ce qui permet à ce dernier de limiter fortement ses débours monétaires.

Ces nouveaux arrangements se nouent autour de relations privilégiées et exclusives avec la catégorie d'ejidataires la mieux pourvue en capital technique (deux tracteurs équipés au minimum) et en main-d'œuvre familiale au vu des surfaces mises en jeu. 
L'émergence extrêmement rapide de cette catégorie d'ejidataires capitalisés (encore absente en 2000) se traduit au sein des ejidos par une différenciation fortement accélérée des systèmes de production agricole, qui repose plus que jamais sur l'accès différencié au capital fixe d'exploitation (puisque c'est lui qui aujourd'hui ouvre l'accès au foncier).

Le cas présenté est relativement extrême. Pour autant, la tendance à l'accumulation foncière par le faire-valoir indirect est très nette pour les ejidataires équipés en matériel agricole. Dans tous les cas, le lien avec le secteur privé et l'agro-industrie est présent et ouvre l'accès à la ressource foncière, même s'il ne s'inscrit pas forcément dans le cadre d'un arrangement tel que celui que nous venons de décrire. En fait, les connexions avec le secteur privé influent sur la croissance de ce type d'exploitations surtout par le biais de traitements différentiels face au crédit de campagne. Ainsi, comme dans le cas exposé, le crédit peut avoir un coût nul, ou alors les "meilleurs » clients vont bénéficier de facilités de paiement, ou encore, on ne leur demandera pas d'acte notarié en cas de location, ce qui est, en principe, indispensable pour se voir accorder un crédit. Mais, dans tous les cas, l'important pour le propriétaire foncier est de conserver l'accès à la récolte qui sera vendue à travers son propre circuit de commercialisation, dont il est généralement propriétaire.

Pour les propriétaires privés, une autre façon d'externaliser la production tout en s'assurant le contrôle de la récolte consiste à prendre en location des parcelles ou des blocs de parcelles au sein des ejidos. Dans ce cas-là, les ejidataires qui cèdent leur parcelle en location perçoivent un loyer et il n'est pas rare qu'une partie du travail agricole soit effectué directement par eux, parfois même avec leur propre matériel agricole. Ils sont bien entendu rémunérés pour ce service, cependant, ils perdent le bénéfice de la récolte qui revient dans ce cas intégralement au "tenancier $»^{12}$. Mais, plus simplement encore, il suffit à ces nouveaux propriétaires fonciers de fournir aux ejidataires semences, pesticides et engrais, de leur louer des machines agricoles, et d'acheter l'intégralité de la récolte à un prix amputé du coût de l'avance aux cultures et du prêt des machines, c'est-à-dire à un prix bien inférieur à celui du marché. Ce que les ejidataires ne peuvent contester puisque à le faire, ils se priveraient d'un accès aux ressources productives et au marché pour lequel aucune garantie, autre que morale, ne leur est demandée. Ces situations de dépendance des ejidataires envers les détenteurs des moyens de production caractérisent tout particulièrement le contexte actuel de production de sorgho dans les terres basses du Moyen-Coatzacoalcos.

\section{Concentration foncière et accélération des processus de différenciation}

L'étude de cas présentée et analysée dans cet article n'a encore ni l'ampleur ni les caractéristiques des phénomènes les plus emblématiques d'appropriation et de concentration foncières dans certains pays du Sud. Cependant, les dynamiques observées dans les terres basses du Sud-Veracruz autour de la diffusion de la céréaliculture moto-mécanisée permettent de mettre en évidence plusieurs évolutions qui apparaissent comme autant de traits communs aux nouvelles formes de production agricole promues dans le cadre de l'agrobusiness et d'une agriculture mondialisée. 
$30 \mathrm{Au}$ sein des ejidos mexicains jouissant d'un certain avantage comparatif - ici agronomique -, on assiste ainsi à une différenciation socio-économique accélérée entre une poignée d'ejidataires capitalisés, bien connectés aux réseaux ouvrant l'accès aux différentes ressources productives, et les autres. Cette évolution est surtout perceptible dans le Tesechoacan, où les processus en cours représentent un cas typique de ce que les réformes de ces quinze dernières années cherchent à promouvoir dans le secteur agricole mexicain : augmentation de la production et de la productivité, implication forte $\mathrm{du}$ secteur privé dans l'impulsion et l'accompagnement du changement, réallocation du foncier vers les structures agricoles " efficientes », meilleur accès aux ressources productives, notamment le crédit agricole, émergence d'exploitations compétitives engagées dans des dynamiques de croissance rapides, etc. Dans un contexte céréalier qui permet particulièrement de faire jouer les économies d'échelle, l'accès au foncier devient un enjeu central pour la majorité des producteurs des terres basses. Dans ce contexte, l'accès différencié au capital fixe d'exploitation apparaît très clairement comme le principal facteur discriminant sur les marchés fonciers, et, en particulier, celui de la location. Sur ce point, le développement des connexions avec le secteur privé et l'agro-industrie profite en premier lieu aux ejidataires détenteurs du matériel agricole.

31 Mais, l'arrivée récente d'investisseurs et de nouveaux propriétaires privés dans les terres basses laisse également entrevoir une tendance à la perte de contrôle du processus de production pour les petits producteurs, contraints de plus en plus d'adopter des itinéraires techniques imposés par l'agro-industrie à travers des contrats de production plus ou moins formels, voire même, de renoncer à tout droit sur leur récolte, dans le cas de contrats moraux passés avec leur "patron ». Pour les petits producteurs, il s'agit de stabiliser un environnement économique incertain en s'assurant un accès aux facteurs de production (matériel agricole, intrants, crédit, etc.) et aux marchés, tandis que pour les détenteurs du capital technique et financier - et foncier de plus en plus - il s'agit surtout de tirer profit de conditions de production particulièrement favorables, liées ici à une bonne fertilité des terres et à la présence d'une main-d'œuvre qualifiée et peu chère. Tout ceci ne préfigure rien de bon pour les exploitations familiales les plus fragiles, qui se trouvent actuellement dans une quasiimpossibilité d'accumulation en capital technique faute de crédits agricoles. Ceux-là participent malgré eux, notamment à travers l'abandon de l'agriculture et les migrations, à une concentration accrue des facteurs de production aux mains d'une catégorie minoritaire d'ejidataires capitalisés d'une part, et d'investisseurs privés d'autre part.

\section{BIBLIOGRAPHIE}

BRAUN J. von and R. MEINZEN-DICK, « Land grabbing by foreign investors in developing countries: risks and opportunities ", IFPRI, Policy Brief [en ligne], n 13, April 2009, consulté en octobre 2011. URL : http://www.ifpri.org/sites/default/files/publications/bp013all.pdf 
COTULA L., S. VERMEULEN, R. LEONARD and J. KEELEY, Land Grab or Development Opportunity? Agricultural Investment and International Land Deals in Africa, London/Rome, FAO/IIED/IFAD, 2009 [en ligne], consulté en octobre 2011.

URL : http://www.ifad.org/pub/land/land_grab.pdf

DEININGER K. et D. BYERLEE, « Rising Global Interest in Farmland. Can It Yield Sustainable and Equitable Benefits?», World Bank Report, 2010 [en ligne], consulté en octobre 2011.

URL : http://siteresources.worldbank.org/INTARD/Resources/ESW_Sept7_final_final.pdf

GRAIN : http://www.grain.org

\section{NOTES}

1. Ce phénomène a d'ailleurs donné lieu à une profusion de rapports et de publications de la part des principaux organismes internationaux de développement, dont K. Deininger et D. Byerlee pour la Banque mondiale (2010), L. Cotula et al. pour la FAO (2009), J. von Braun et R. MeinzenDick pour l'IFPRI (2009), pour ne citer que ceux-ci. Dans un autre registre, plus critique, de nombreuses ONG s'élèvent contre cette "ruée " sur les terres arables des pays du Sud (voir l'organisation GRAIN) et des organisations de paysans font entendre leur voix (voir l'appel de Dakar en 2011).

2. M. Merlet, «Les phénomènes d'appropriation à grande échelle des terres agricoles dans les pays du Sud et de l'Est ", Études foncières, nº 142, novembre-décembre 2009.

3. L'ejido est une forme juridique de propriété sociale issue de la réforme agraire mexicaine (proclamée en 1915). Le terme se réfère à la fois à l'ensemble des terres attribuées par principe de dotation à un groupe de paysans (qu'on appelle ejidataires), et à ce groupe social constitué.

4. Nous préférons utiliser le terme de concentration foncière plutôt que ceux d'appropriation ou d'accaparement qui suggèrent un certain flou quant à la nature des droits fonciers acquis. Ce n'est pas le cas au Mexique où les droits fonciers sont bien établis.

5. PROCEDE pour Programa de certificación de derechos ejidales y titulación de solares

6. D'après les données de l'Institut national de statistique et de géographie (INEGI) publiées en 2007. Le PROCEDE, officiellement terminé en 2006, se déroulait en deux étapes soumises chacune à l'acceptation de l'assemblée ejidale. La première étape consistait en une inscription au cadastre du parcellaire ejidal et a abouti à la délivrance de certificats individuels de droit agraire pour chaque parcelle mesurée. La seconde, appelée Dominio Pleno, permettait la privatisation partielle ou totale de l'ejido et donnait lieu à la remise de titres de propriété. Dans l'immense majorité des cas, seule la première phase du PROCEDE a été acceptée (entre 1991 et 2007, seuls $19 \%$ des ejidos ont opté pour une privatisation partielle ou totale de leur territoire).

7. Voir J. Fox et L. Haight, Subsidios para la desigualdad: las políticas públicas del maíz en México a partir del libre comercio, México, Woodrow Wilson International Center for Scholars, 2010, et aussi, E. Léonard, A. Quesnel et E. Velásquez (coords), Políticas y regulaciones agrarias. Dinámicas de poder y juegos de actores en torno a la tenencia de la tierra, México, CIESAS/IRD, 2003.

8. Cas du Pronamat (Programa nacional de maíz de alta tecnología), programme de transfert technologique dans les zones d'agriculture irriguée et celles bénéficiant d'avantages comparatifs, qui reposait sur la diffusion de nouvelles techniques culturales et sur la décentralisation des services d'assistance technique. Coordonné dans la région du Tesechoacan, à partir de 1990, par deux institutions publiques, le FIRA (Fideicomiso en relación con la agricultura) et l'INIFAP (Instituto nacional de investigación forestal y agropecuaria), le Pronamat a pris la forme d'un projet de diffusion du semis direct mécanisé et d'incitation à l'utilisation de variétés commerciales de maïs à haut rendement. 
9. V. Brun, «Secteur privé et céréaliculture familiale dans le Mexique du libre-échange. Une étude dans les terres basses du Sud-Veracruz ", Économie rurale, n 303-304-305, janvier-mai 2008 : 90-107.

10. Voir en particulier J.-P. Colin, C. Blanchot, E. Vásquez et H. Navarro, «Réorganisations productives et pratiques foncières sur un périmètre irrigué ", in J.-P. Colin (ed.), Figures du métayage : étude comparée de contrats agraires (Mexique), Paris : IRD Éditions, collection «À travers champs », $2003: 111-157$; et E. Léonard et B. Losch, «L'insertion de l'agriculture mexicaine dans le marché nord-américain: changements structurels, mutation de l'action publique et recompositions de l'économie rurale et régionale", communication au colloque Intégrations régionales et stratégies de développement, RINOS - CEIM UQAM, Montréal, 1-3 juin 2005.

11. La loi de 1991 stipule d'ailleurs que les cessions définitives ne peuvent s'effectuer qu'entre les membres d'un même ejido dans le cas où ce dernier conserverait son statut juridique. Ces transactions requièrent par ailleurs une autorisation préalable qui doit être votée en assemblée et validée par un minimum de $75 \%$ de l'ensemble des ejidataires. Enfin, aucun membre de l'ejido ne peut détenir individuellement plus de $5 \%$ de la superficie totale de l'ejido... ce qui n'empêche pas d'avoir recours à un ou plusieurs prête-noms pour outrepasser cette règle. Les achats par des personnes ou sociétés privées extérieures sont en principe illégaux, mais rien n'interdit à un ejidataire de céder de façon " gratuite " sa parcelle à un acheteur potentiel...

12. Des cas similaires ont été décrits dans d'autres régions du monde et notamment en France (voir H. Cochet, «Vers une nouvelle relation entre la terre, le capital et le travail », Études foncières, $\mathrm{n}^{\circ} 134$, juillet-août 2008.

\section{RÉSUMÉS}

Depuis le début des années 1990, l'agriculture mexicaine connaît de profondes restructurations qui s'inscrivent dans un ensemble de réformes visant à accélérer l'insertion compétitive des exploitations sur les marchés agricoles, notamment dans le cadre de l'Accord de libre-échange nord-américain liant le Mexique, les États-Unis et le Canada depuis le $1^{\mathrm{er}}$ janvier 1994. Dans le domaine foncier, la révision, en 1991, de l'article 27 de la Constitution mexicaine a signifié la fin des redistributions foncières - à l'origine de la formation des ejidos -, et la réintégration aux marchés fonciers des terres distribuées dans le cadre de la réforme agraire (autorisation de leur cession définitive et/ou temporaire et de leur mise en gage). En instaurant par ailleurs un cadre institutionnel propice à l'émergence de marchés fonciers dans les ejidos via un important programme de titrage individuel des droits fonciers, la réforme devait contribuer à la mise en place de nouvelles dynamiques productives, en forçant la réallocation des ressources productives, notamment la terre, vers les structures les plus compétitives. Les concentrations foncières aux mains des plus " productifs » sont donc désormais possibles et même souhaitées, et cela, en totale contradiction avec le Code agraire qui interdit toujours la grande propriété au Mexique.

Les questions foncières liées aux transformations productives et au changement institutionnel sont traitées ici à partir d'une étude de cas conduite dans différents ejidos des terres basses du Sud-Veracruz (bassins du Tesechoacan et du Moyen-Coatzacoalcos), où l'on observe depuis le milieu des années 1990 un développement important de la production mécanisée de maïs et de sorgho destinée aux marchés. Il s'agit notamment, après la révision constitutionnelle de 1991, de dresser un état des lieux des dynamiques foncières liées à la diffusion de la céréaliculture 
commerciale, lesquelles se manifestent par un accroissement marqué de la demande sur les marchés fonciers internes et externes aux ejidos. Il s'agit aussi de montrer comment l'accès des petits producteurs aux différentes ressources productives (terre, intrants et capital technique) s'organise aujourd'hui à différentes échelles, au sein des ejidos d'une part, mais également autour de l'intervention de nouveaux acteurs issus du secteur privé (agro-industriels, entrepreneurs et opérateurs agricoles, fournisseurs d'intrants et de services, etc.). Que ces derniers prennent part ou non à un récent phénomène d'acquisitions foncières - qui n'a toutefois ni l'ampleur ni les caractéristiques des grandes appropriations foncières ayant actuellement lieu dans certains pays du Sud -, ils jouent un rôle de plus en plus central dans les stratégies d'expansion des petites exploitations familiales et dans les processus locaux d'intégration commerciale. Dans le cas du bassin du Tesechoacan, le modèle d'intégration impulsé par les acteurs privés s'apparente à une forme d'agriculture sous contrat (sur le modèle développé par l'agrobusiness), tandis que dans celui du Moyen-Coatzacoalcos, le schéma fonctionne avant tout sur la base de relations personnalisées de type clientéliste. Dans les deux cas toutefois, les logiques d'intervention de ces nouveaux acteurs privés s'appuient sur un contrôle accru des exploitations familiales, tant en amont (accès aux ressources productives) qu'en aval (mise en marché) de la production. Par ailleurs, la présence du secteur privé bénéficie en premier lieu à une catégorie émergente d'ejidataires "capitalisés ", dont le rapprochement avec ces nouveaux acteurs se construit autour de la prise de terres en location. L'une des conséquences est une différenciation socioéconomique accélérée au sein des ejidos. Au final, l'analyse des processus productifs en cours dans les terres basses du Sud-Veracruz permet de mettre en évidence une tendance à l'accumulation et à la concentration de terres, aux mains d'une catégorie minoritaire d'ejidataires bien dotés en capital technique d'une part, et d'entrepreneurs privés contrôlant les accès aux ressources productives et aux marchés d'autre part.

Since the early 1990s, Mexican agriculture has undergone major restructuration as part of reforms aiming to help the country's farms take their place on agricultural markets. This is especially true with respect to the North American Free Trade Agreement, signed on 1 January 1994 by the United States, Canada and Mexico. As far as land is concerned, Article 27 of the Mexican Constitution was revised in 1991 to put a stop to land redistributions, linked to the creation of ejidos, and reintegrate land distributed as a result of the agrarian reform into property markets (in particular, it authorised permanent and temporary land transfers and using land as collateral). In addition, the reform introduced a new system of land title to ejidos in order to encourage emerging property markets. This was to support new forms of productivity by forcing the reallocation of productive resources (land in particular) to more competitive structures. Allowing the most productive to amass land was now possible and even desirable, despite contradictory provisions prohibiting large estates in the Agrarian Code. This study focuses on land issues linked to productive transformations and institutional change by observing several ejidos in the south Veracruz lowlands (the Tesechoacan River basin and middle reaches of the Coatzacoalcos River). Since the mid 1990s, mechanised production of corn and sorghum crops for the market has increased considerably. The study will examine the changes affecting land following the 1991 constitutional revision and the spread of commercial grain farming. In particular, the study will look into the increase in demand for land inside and outside ejidos. It will also show how small producers' access to productive resources (land, inputs and technical capital) is organised on several levels - within ejidos, but also with new private sector actors (industrial farmers, companies, agricultural operators, and input and service suppliers, etc.). Whether or not the latter take part in the recent trend of land acquisitions - on a much smaller scale than the large-scale land acquisitions taking place in other developing countries they play an increasingly important role in the expansion strategies of small family farms and local commercial integration processes. In the Tesechoacan basin, the integration model pushed 
by private sector actors resembles a form of contract farming (based on the agribusiness model). In the middle Coatzacoalcos River, it operates via a clientelistic system of personal relationships. However, in both cases, these new private sector actors intervene to increase their control over family farms, both upstream (by controlling productive resources) and downstream (by getting produce to market). Moreover, the presence of the private sector benefits a new category of "capitalised" ejidatarios, who build relationships based on renting land. Consequently, there are growing socio-economic differences between ejidos. Finally, by analysing the productive processes at work in the south Veracruz lowlands, the study highlights the increasing accumulation and concentration of land by two groups: ejidatarios with significant technical capital on the one hand, and private entrepreneurs controlling access to productive resources and the market on the other.

\section{INDEX}

Index géographique : Mexique

Mots-clés : agriculture familiale, secteur privé, marché foncier, concentration foncière

Keywords : family farming, private sector, property market, land concentration, Mexico

\section{AUTEUR}

\section{VIRGINIE BRUN}

Agroéconomiste, elle est chercheuse associée au Centre d'études mexicaines et centraméricaines (CEMCA - UMIFRE 16 CNRS-MAEE), à Mexico, où elle a été allocataire de 2007 à 2009. Elle termine actuellement une thèse de doctorat en agriculture comparée (AgroParisTech / IRD) sur les dynamiques d'insertion marchande des exploitations familiales dans l'État de Veracruz au Mexique. Ses recherches portent de façon générale sur les processus de développement des agricultures familiales du Sud et plus particulièrement sur les conditions de ce développement dans un contexte de libéralisation économique et de mondialisation des échanges.

Adresse postale : 39, rue Adolphe Cadéot 32500 Fleurance

virginia.brun@gmail.com 